\title{
The effects of Y-shaped conchal cartilage transplantation on the correction of nasal deformity secondary to cleft lip and its influence on mental health
}

\author{
LING LIU $^{1}$, WEI WANG ${ }^{2}$, LIN YANG ${ }^{2}$ and HONGCHUANG ZHANG ${ }^{3}$ \\ Departments of ${ }^{1}$ Cosmetology, ${ }^{2}$ Plastic Surgery and ${ }^{3}$ Stomatology, \\ The First People's Hospital of Xuzhou, Xuzhou, Jiangsu 221002, P.R. China
}

Received April 27, 2017; Accepted July 17, 2017

DOI: $10.3892 /$ etm.2017.4994

\begin{abstract}
Patients who undergo corrective surgery for cleft lip are known to be at risk for subsequently developing secondary nasal deformity. The aim of this study was to investigate the effects of Y-shaped conchal cartilage transplantation on the correction of nasal deformity secondary to cleft lip and its influence on mental health. Sixteen patients with nasal deformity secondary to cleft lip admitted to The First People's Hospital Xuzhou (Xuzhou, China) from February 2014 to February 2015 were selected for the study. Conchal cartilage was taken from the patients and made into a Y-shaped stent, which was then transplanted into the nasal columella. The surgical outcomes and patient satisfaction were evaluated and HADS was used to compare the preoperative and postoperative mental health. After the affected side of the nose was corrected, the nasal profiles of 12 patients were significantly improved, the height of nasal columella and nostril was significantly increased, the nasal base and breadth were significantly shorter than those before operation and the nose wing-nasal breadth index on the affected side was significantly increased compared to that before operation and the differences were statistically significant $(\mathrm{P}<0.05)$. The satisfaction of each index after the operation was more than $90 \%$. The mental health of patients after operation was notably improved compared to before operation and the difference was statistically significant $(\mathrm{P}<0.05)$. After the correction of secondary nasal deformity to cleft lip through Y-shaped conchal cartilage transplantation, the shape of the nasal tip was stable without the recurrence of deformities or distortion on the donor site of the auricle. Y-shaped conchal cartilage transplantation is an
\end{abstract}

Correspondence to: Dr Hongchuang Zhang, Department of Stomatology, The First People's Hospital of Xuzhou, 19 Zhongshan North Road, Xuzhou, Jiangsu 221002, P.R. China

E-mail: hczhang21@163.com

Key words: conchal cartilage, cleft lip, nasal deformity, anxiety, depression ideal treatment method for the moderate and severe secondary nasal deformity to cleft lip and effective perioperative mental intervention can improve the patient's mental health status, an outcome that is worth popularizing in clinical applications.

\section{Introduction}

Cleft lip, also known as 'harelip', refers to a rupture of the upper lip, which is a clinically common type of congenital malformation. The ratio of male patients to female patients is $1.5: 1$ and its presence can affect the localized development of muscle, skin, bone and cartilage $(1,2)$. Surgical treatment is the predominant repair method for cleft lip, though about $80 \%$ of patients will suffer from secondary nasal deformity after the repair (3). Nasal deformity secondary to cleft lip is usually characterized by nasal columella deviation, low and collapsing nasal tip on the affected side and low nose wing and nasal septum distortion, leading to a sunken nasal base that is wider than that on the unaffected side. These and other potential deformities can become an issue for patients that cannot be ignored and so necessitate their correction. Compared with the initial correction of lip deformity, the correction of nasal deformity secondary to the cleft lip, especially nasal deformity secondary to unilateral cleft lip, is much more difficult. Therefore, it is one of the more complicated problems in plastic surgery $(4,5)$. The majority of patients often fail to receive complete correction due to the substandard or delayed treatment, seriously influencing their work and life, so these patients generally suffer from serious psychological problems (6). In the surgical correction, patients usually experience serious anxiety and depression due to apprehension of the operative results and so perioperative psychological intervention is needed in order to facilitate a smooth operation and rapid postoperative recovery (7). In this study, 16 patients with secondary nasal deformity to cleft lip received the Y-shaped conchal cartilage transplantation and the surgical outcomes were observed.

\section{Materials and methods}

General information. Sixteen patients with nasal deformity secondary to cleft lip admitted to The First People's Hospital of 
Xuzhou (Xuzhou, China) from February 2014 to February 2015 were selected for the study. The conchal cartilage was taken from the patients and made into a Y-shaped stent which was then transplanted into the nasal columella. The clinical manifestations of patients included inadequate lip height, uneven vermilion border, asymmetry of bilateral nostrils, nasal columella which deflected to the affected side, wider nasal base and collapsing nasal tip. All of the patients signed an informed consent voluntarily. Exclusion criteria: i) Patients with coagulation dysfunction; ii) patients with mental illness or immune system diseases; and iii) patients allergic to narcotic drugs. The general information of the research subjects is shown in Table I. This study was approved by the Ethics Committee of the First People's Hospital of Xuzhou. Signed written informed consents were obtained from the patients and/or guardians.

\section{Methods}

Preoperative preparation. Before the operation, all patients received routine examination and a coagulation function examination. Patients had a rational and nutritious diet, laying the foundation for a functional postoperative recovery. According to the varying personalities of each patient, one-on-one personalized psychological counseling was provided, so as to ease the tension, anxiety, depression and other unhealthy emotions before the operation. Each evaluation index of the operation was measured and evaluated before the operation. On the day of the operation, the patients' oral cavity and nasal cavity were cleaned, in order to avoid infection and patients fasted for $4 \mathrm{~h}$ before the operation.

Anesthesia plan. The 16 patients received general anesthesia by intubation and 5\% lidocaine was injected during operation for bilateral infraorbital nerve block anesthesia and local infiltration, so as to minimize intraoperative hemorrhage.

Incision design. The flying-bird-shaped incision was designed in the nose wing and the nasal columella. The skin of nasal vestibule was incised. The skin of nose wing was completely separated and the nasal alar cartilage was fully released and thoroughly separated from the surrounding tissue adhesion to make it fully exposed. Part of the cartilage of medial margin was then removed to narrow the nose and the surgeons decided whether or not to cut off the nose wing according to the collapse of nose wing. At the same time, the contractual nasal muscles were divided.

Autologous conchal cartilage. An arc-shaped incision was made in the dorsal cavity of concha and the subcutaneous tissue was cut thoroughly to the surface of the conchal cartilage. The unilateral or bilateral conchal cartilage with periosteum were taken according to the requirements of the operation (approximately $2.5 \times 1.2 \mathrm{~cm}$ ) and placed on standby in a solution containing 240,000 units of gentamicin in $250 \mathrm{ml}$ normal saline. After hemostasis was achieved, the skin incision was sutured with pressure dressing (Fig. 1).

Conchal cartilage transplantation. The standby autologous conchal cartilage was made into the Y-shaped stent and the lower end of the stent was transplanted between the medial crura of the bilateral greater alar cartilage and sutured in the shape of a U. The upper ends of the Y-shaped stent were fixed on the nasal fornix, with the conchal cartilage in a T-shape. The top of the bilateral nasal alar cartilage was suspended using silk thread, making it the same horizontal height as both wings of the Y-shaped stent. The scar flap was made by cutting along the lip scar edge to the fascia layer (from vermilion border to nasal base). Its epidermis was removed and filled below the lateral crus of nose wing on the affected side and in the nasal base. Finally, the scar was sutured after hemostasis was achieved.

Postoperative nursing. After the operation, the incision was observed periodically to check for hemorrhage or obvious oozing of blood and any evidence of bleeding was addressed in a timely manner. The incision was kept clean and dry and antibiotic ointment was applied for conventional antiinfection care. Oral care was conducted twice per day and the silicone-tube nostril stent was worn for one month. Doctors observed whether there was any diastasis or shifting and took measures to correct it as necessary. Postoperative follow-up was conducted for 12 months to observe the recovery condition.

\section{Evaluation indexes}

Evaluation criteria of nasal deformity. i) Mild: The nasal tip shape was normal, the nose wing was collapsed slightly, the nasal base was not obviously collapsed and the nasal columella was slightly skewed. ii) Moderate: The nasal tip shape was abnormal but not obviously, the nose wing was collapsed moderately, the nasal base was obviously collapsed and the nasal columella was slightly skewed and small. iii) Severe: The nasal tip shape was obviously abnormal, the nose wing was collapsed significantly and the nasal base was obviously collapsed and accompanied by an oronasal fistula.

Quantitative evaluation of operation result. The height of the nasal columella, the width of the nasal base on the affected side and the nostril height and nose breadth (distance between the furthest lateral points of bilateral nose wings divided by two) on the affected side were measured before and after surgery. The harmonious degree between the nasal height and the nasal base breadth on the unaffected and the affected sides was also evaluated, using the nose wing length-nose breadth index, as follows: nose wing length/nose breadth x $100 \%$.

Patients' mental health. Patients' mental health was evaluated using the Hospital Anxiety and Depression Scale (HADS). The scale included 14 questions 7 for the evaluation of depression and 7 for anxiety. Each question had four possible grades (ranging from 0 to 3 ), according to the degree. The grades for each question were added to determine the scores. Scores of 7-9 indicated suspected symptoms and scores $>9$ indicated anxiety or depression.

Evaluation of patient satisfaction. Patient satisfaction was evaluated by one operator, one nurse, the patient and 2 senior plastic surgeons after the operation and focused on six facial aspects: Symmetry of nasal base width, nostril shape, reconstruction of agger nasi, recovery of nasal columella height, symmetry of nose wing and the centering of nasal tip and nasal columella. Evaluation criteria were as follows: i) Very 
Table I. General information on the research subjects.

\begin{tabular}{lc}
\hline Item & Patients, $\mathrm{n}=60$ \\
\hline Male, no. (\%) & $10(62.50)$ \\
Female, no. (\%) & $6(37.50)$ \\
Age (years) & $16-38$ \\
Average age (years) & $24.36 \pm 4.49$ \\
Degree of deformity, no. (\%) & \\
Moderate nasal deformity & $11(68.75)$ \\
Severe nasal deformity & $5(31.25)$ \\
\hline
\end{tabular}

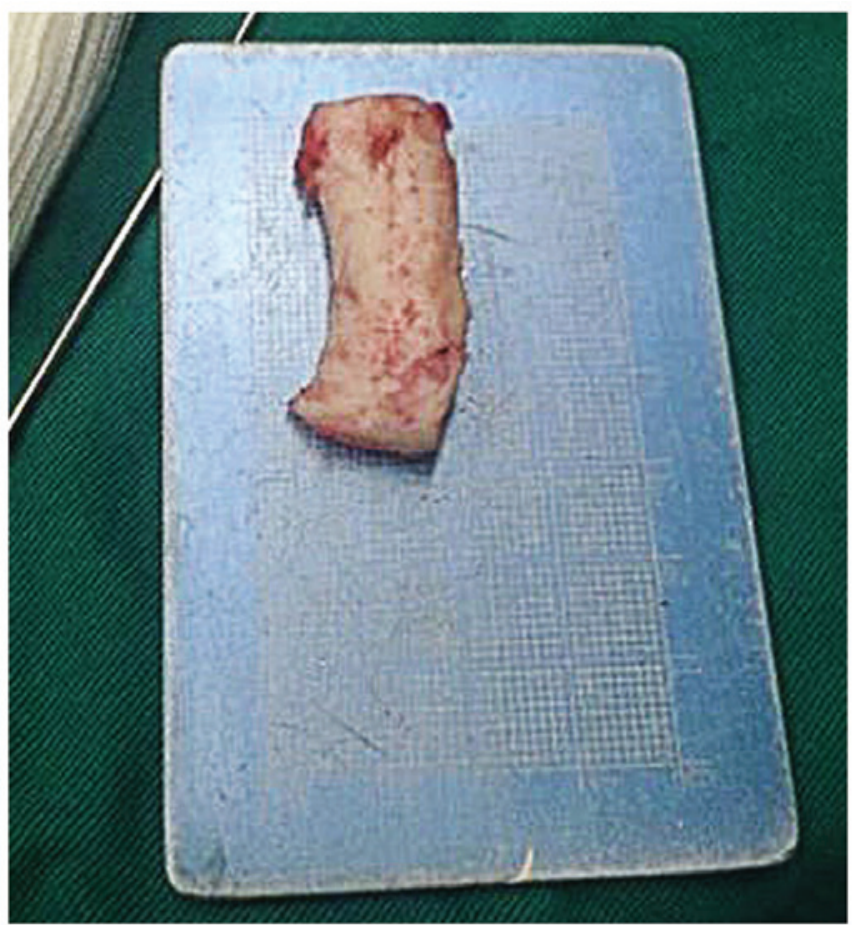

Figure 1. Autologous conchal cartilage.

satisfactory: All 5 people were satisfied with the surgical outcome; ii) satisfactory: 3-4 people were satisfied with the surgical outcome; and iii) not satisfactory: Fewer than 3 people were satisfied with the surgical outcome. Total satisfaction $=$ very satisfactory + satisfactory.

Statistical analysis. SPSS 19.0 software (IBM SPSS, Armonk, NY, USA) was used for all data processing and statistical analyses. Measurement data are presented as mean \pm standard deviation and t-test was used to compare the values between two groups. $\mathrm{P}<0.05$ was considered to indicate a statistically significant difference.

\section{Results}

The comparison of measurement results of the nasal columella on the affected side, nostril height, nasal base and nose breadth of the 16 patients before and after operation are shown (Figs. 2-5). The heights of the nasal columella

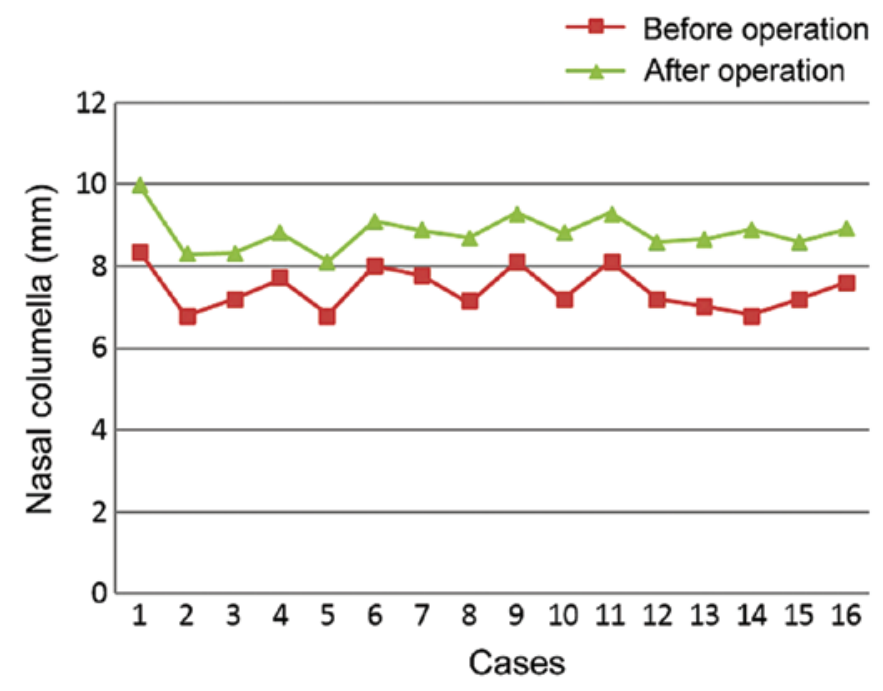

Figure 2. Measurement of nasal columella of 16 patients before and after the operation. Each square symbol represents the nasal columella measurement $(\mathrm{mm})$ of a patient before the operation, with the triangle symbol directly above representing the same patient after the operation.

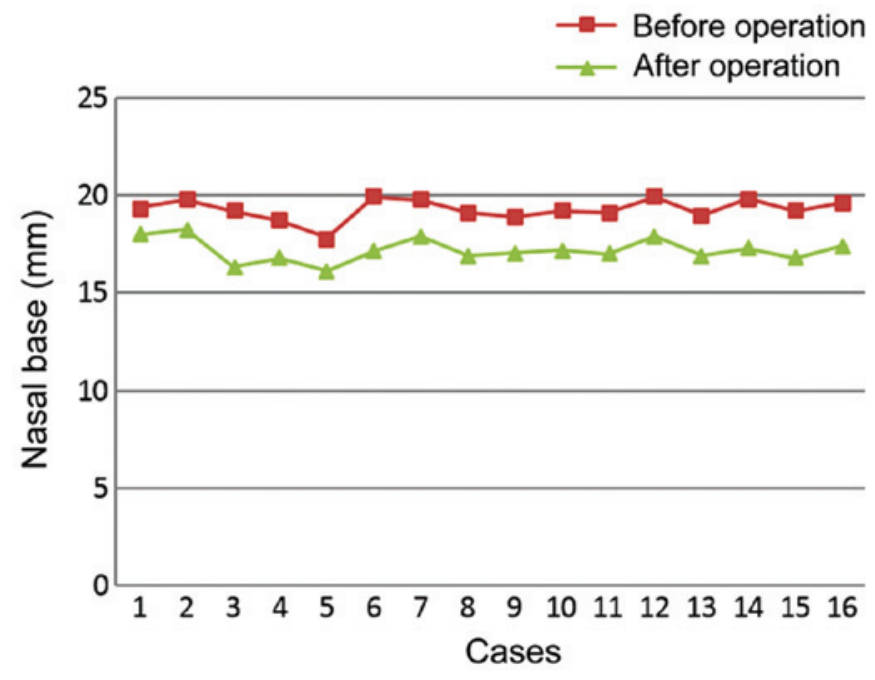

Figure 3. Measurement of nasal base of 16 patients before and after the operation. Each square symbol represents the nasal base measurement (mm) of a patient before the operation, with the triangle symbol directly above representing the same patient after the operation.

and the nostril were significantly increased compared to that before the operation and the nasal base and nasal breadth were significantly shorter than before operation. Each of the differences were statistically significant $(\mathrm{P}<0.05)$ (Table II).

Comparison of harmonious degree. The harmonious degree was compared between the nasal height and nose wing width on the unaffected and the affected sides of the patients. After the surgical correction, the nose wing length-nose breadth index on the affected side was increased $(\mathrm{P}<0.05)$ and the indices on the unaffected and affected sides were almost the same (Table III).

Comparison of HADS scores. After the surgical correction, the HAS and HDS scores of patients were significantly lower 
Table II. Comparison of affected sides before and after the operation $(\mathrm{mm})$.

\begin{tabular}{lcccc}
\hline Item & $\begin{array}{c}\text { Before } \\
\text { operation }\end{array}$ & $\begin{array}{c}\text { After } \\
\text { operation }\end{array}$ & t-test & P-value \\
\hline $\begin{array}{l}\text { Height of nasal } \\
\text { columella }\end{array}$ & $7.06 \pm 0.48$ & $8.86 \pm 0.57$ & 9.662 & $<0.0001$ \\
$\begin{array}{l}\text { Width of } \\
\text { nasal base }\end{array}$ & $19.73 \pm 0.38$ & $17.43 \pm 0.33$ & 18.280 & $<0.0001$ \\
$\begin{array}{l}\text { Height of } \\
\text { nostril }\end{array}$ & $10.16 \pm 0.23$ & $12.75 \pm 0.43$ & 21.245 & $<0.0001$ \\
$\begin{array}{l}\text { Nasal breadth } \\
21.53 \pm 0.58\end{array}$ & $18.83 \pm 0.31$ & 16.422 & $<0.0001$ \\
\hline
\end{tabular}

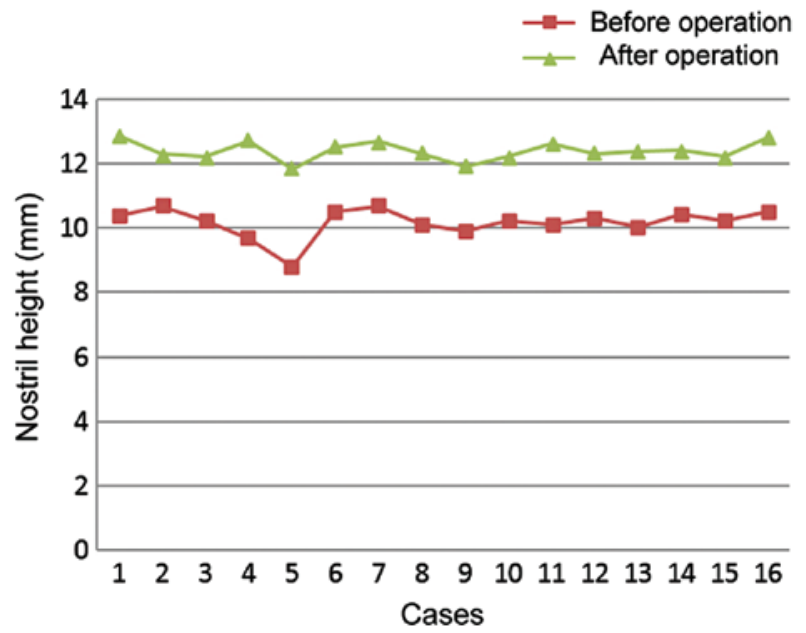

Figure 4. Measurement of nostril height of 16 patients before and after the operation. Each square symbol represents the nostril height measurement $(\mathrm{mm})$ of a patient before the operation, with the triangle symbol directly above representing the same patient after the operation.

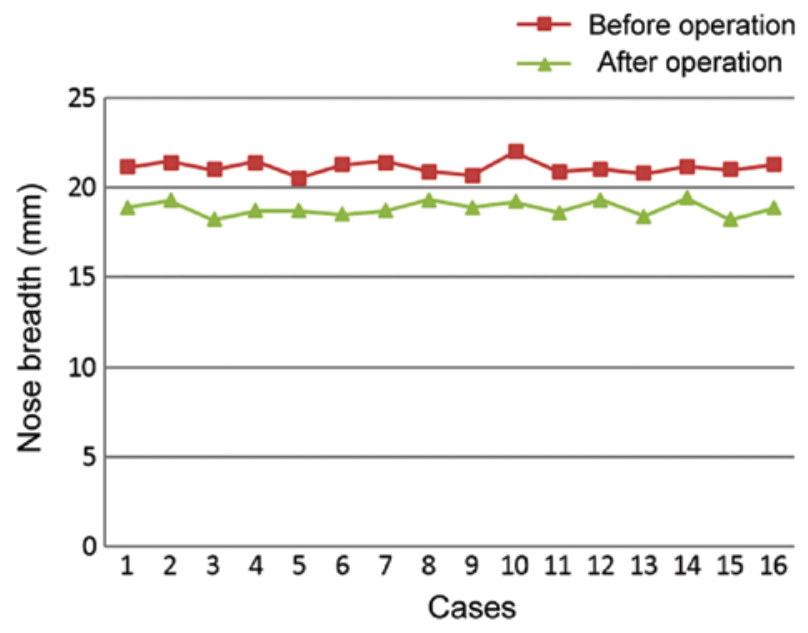

Figure 5. Measurement of nose breadth of 16 patients before and after the operation. Each square symbol represents the nose breadth measurement $(\mathrm{mm})$ of a patient before the operation, with the triangle symbol directly above representing the same patient after the operation.

than those before operation and the differences were statistically significant $(\mathrm{P}<0.05)$ (Table IV).
Comparison of patient satisfaction. The overall patient satisfaction of the operation for each index was above $90 \%$ (Table V).

\section{Discussion}

Causes of cleft lip and secondary nasal deformity. There are many pathogenic factors for cleft lip, including the following: i) Endocrine factors: Abnormal hormone secretion or adverse drug effects lead to excessive hormone levels in the body. For example, excess adrenaline, or long-term use of dexamethasone or other drugs are likely to lead to the occurrence of cleft lip (8); ii) infection of pregnant women: Viral infection that occurs in the mother's body in the first trimester of pregnancy, such as the pestivirus infection, may cause fetal malformations, thus forming the cleft lip (9); iii) nutrition factors: Nutritional deficiency in the mother's body during pregnancy, especially the lack of vitamins, folic acid and pantothenic acid, can also lead to cleft lip; iv) chemical and physical factors: During the first trimester of pregnancy, if pregnant women take certain drugs that result in adverse side effects, have frequent exposure to radiation, microwaves or other harmful physical interference, or drink alcohol or smoke, the risk of malformations such as cleft lip is increased (10); and v) genetic factors: If parents or other relatives suffer from cleft lip, the fetus may also suffer from the cleft lip due to genetic factors (11). There are also numerous reasons for nasal deformity secondary to cleft lip, including mainly: i) Affected by cleft lip, the existing tissue will suffer from shift or hypoplasia, causing nasal alar cartilage distortion and nasal deformity; ii) after corrective surgery for cleft lip, the subsequent growth and development may also cause secondary nasal deformity depending on the age at the time of operation; and iii) other factors, such as the training level of surgeons and different surgical procedures, may also cause an increased risk of secondary nasal deformity, so the stage-II repair is recommended for patients to ameliorate this risk $(12,13)$.

Treatment of nasal deformity secondary to cleft lip. Nasal deformity secondary to cleft lip is treated mainly through surgery. Although there are many surgical methods, it remains a challenge to achieve completely satisfactory results, so the correction of nasal deformity secondary to cleft lip is a difficult problem in plastic surgery (14). Most researchers believe that premature corrective surgery will influence the development of nasal alar cartilage, such that the nose will still suffer from secondary deformity after full development. Therefore, it is appropriate to correct cleft lip of patients after the age of 16 years, so that the nose tip shape can remain stable and the incidence of recurrent deformity can be reduced (6). For the correction of mild nasal deformity secondary to cleft lip, a guided needle is often buried and the tissue is displaced. However, this just repairs the soft tissue and it will be affected by the elasticity of nasal alar cartilage, resulting in the recurrence of nasal deformity after operation. Therefore, moderately severe nasal deformity is more often corrected by cartilage transplantation, which achieves an ideal long-term effect (15).

Transplantation material can be categorized as autograft or allograft, the latter of which can readily cause shifting, infection and a rejection reaction. In contrast, an autograft 
Table III. Comparison of nose wing lengt-nose breadth index before and after the operation.

\begin{tabular}{lcrr}
\hline Item & Before operation & After operation & t-test \\
\hline $\begin{array}{l}\text { Nose wing length-nose breadth index } \\
\text { (affected side) }\end{array}$ & $49.06 \pm 3.48$ & $54.46 \pm 2.57$ & 4.993 \\
$\begin{array}{l}\text { Nose wing length-nose breadth index } \\
\text { (unaffected side) }\end{array}$ & $51.73 \pm 1.38$ & $54.83 \pm 1.33$ & 6.470 \\
\hline
\end{tabular}

Table IV. Comparison of HADS scores of patients before and after the operation.

\begin{tabular}{lcccc}
\hline Item & $\begin{array}{c}\text { Before } \\
\text { operation }\end{array}$ & $\begin{array}{c}\text { After } \\
\text { operation }\end{array}$ & t-test & P-value \\
\hline HAS & $9.45 \pm 2.34$ & $5.69 \pm 2.38$ & 4.506 & 0.0001 \\
HDS & $9.48 \pm 3.35$ & $5.83 \pm 2.43$ & 3.528 & 0.0014 \\
\hline
\end{tabular}

HADS, Hospital Anxiety and Depression Scale; HAS, Hospital Anxiety Scale; HDS, Hospital Depression Scale.

Table V. Comparison of the results on the satisfaction survey on operative outcomes.

\begin{tabular}{|c|c|c|c|c|}
\hline Item & $\begin{array}{c}\text { Very } \\
\text { satisfactory }\end{array}$ & Satisfactory & $\begin{array}{c}\text { Not } \\
\text { satisfactory }\end{array}$ & $\begin{array}{c}\text { Overall } \\
\text { satisfaction }\end{array}$ \\
\hline Recovery of nasal columella height & $12(75.00)$ & $3(18.75)$ & $1(6.25)$ & $15(93.75)$ \\
\hline Nostril shape & $11(68.75)$ & $4(25.00)$ & $1(6.25)$ & $15(93.75)$ \\
\hline Symmetry of nose wing & $13(81.25)$ & $2(12.50)$ & $1(6.25)$ & $15(93.75)$ \\
\hline Reconstruction of agger nasi & $12(75.00)$ & $3(18.75)$ & $0(0.00)$ & $16(100.00)$ \\
\hline Symmetry of nasal base width & $13(81.25)$ & $2(12.50)$ & $1(6.25)$ & $15(93.75)$ \\
\hline Centering of nasal tip and nasal columella & $11(68.75)$ & $4(25.00)$ & $1(6.25)$ & $15(93.75)$ \\
\hline
\end{tabular}

Values indicate the number of cases in each category with percentage of total cases in paranetheses. Overall satisfaction refers to cases that are either very satisfactory or satisfactory.

can cause fewer complications, so it is widely used in clinical applications (16). Autologous materials include nasal septum cartilage, autologous costal and conchal cartilage, among others. The nasal septum cartilage has a small volume and the building and support of a cartilaginous stent are insufficient. These factors, combined with the high operation difficulty, make it not suitable for clinical popularization (17). Costal cartilage is sufficient and it is easy to build the required stent, but fine carving is needed, so the operation is expensive, which is not acceptable for patients with poor economic status (18). Conchal cartilage is easily obtained, it can meet the basic surgical requirements and the operation cost is low, so it is welcomed by most patients (19).

Advantages of $Y$-shaped conchal cartilage transplantation. In this study, the autologous conchal cartilage of patients was shaped and made into a Y-shaped conchal cartilage stent used in corrective surgery and satisfactory results were obtained. The study results showed that, after corrective surgery, the nose shape was significantly improved, the nasal base and nose breadth were obviously shorter than before the operation, the nasal columella and nostril height were significantly higher than those before operation and the nose wing length-nose breadth index on the affected side was notably increased compared to that before the operation $(\mathrm{P}<0.05)$. The arc-shaped incision on the dorsal cavity of the auricular concha is hidden, and its influence on the appearance is not obvious after healing without the outer ear deformity, so it can reduce the psychological burden of patients. At the same time, perioperative psychological interventions can ease patients' anxiety and improve their mental health status. Y-shaped conchal cartilage transplantation has a reduced risk of trauma with a lower incidence of postoperative complications. Conchal cartilage also has a natural mechanical structure and both wings of the stent can provide strong support for nasal alar cartilage, thus ensuring that the nose is not likely to suffer shift. The satisfaction evaluation of the surgical outcomes from this study show that the satisfaction of each index is above $90 \%$.

In conclusion, Y-shaped conchal cartilage transplantation is an ideal treatment method for nasal deformity secondary to cleft lip. The roundness of nose wing can be corrected, the 
nasal tip can be raised significantly and the nasal tip shape can be stabilized without recurrent deformity after the operation, which are surgical outcomes worth popularizing in clinical applications.

\section{References}

1. Nyberg J, Peterson P and Lohmander A: Speech outcomes at age 5 and 10 years in unilateral cleft lip and palate after one-stage palatal repair with minimal incision technique-a longitudinal perspective. Int J Pediatr Otorhinolaryngol 78: 1662-1670, 2014.

2. Pitak-Arnnop P, Hemprich A, Dhanuthai K, Yildirim V and Pausch NC: Panel and patient perceptions of nasal aesthetics after secondary cleft rhinoplasty with versus without columellar grafting. J Craniomaxillofac Surg 39: 319-325, 2011

3. Li J, Shi B, Liu K and Zheng Q: A preliminary study on the hard-soft tissue relationships among unoperated secondary unilateral cleft nose deformities. Oral Surg Oral Med Oral Pathol Oral Radiol 113: 300-307, 2012.

4. Agarwal R and Chandra R: Alar web in cleft lip nose deformity: Study in adult unilateral clefts. J Craniofac Surg 23: 1349-1354, 2012.

5. Wang H, Fan F, You J and Wang S: Correction of unilateral cleft lip nose deformity using nasal alar rim flap. J Craniofac Surg 23: $1378-1381,2012$

6. Mokal NJ and Juneja M: Secondary bilateral cleft lip-nose deformity correction by rhinoplasty with simultaneous Abbe flap. Indian J Plast Surg 47: 20-24, 2014.

7. Nakamura N, Okawachi T, Nozoe E, Nishihara $K$ and Matsunaga K: Three-dimensional analyses of nasal forms after secondary treatment of bilateral cleft lip-nose deformity in comparison to those of healthy young adults. J Oral Maxillofac Surg 69: e469-e481, 2011.

8. Akin MA, Kurtoğlu S, Sarici D, Akin L, Hatipoğlu N, Korkmaz L, Günes T, Oztürk MA and Akçakus M: Endocrine abnormalities of patients with cleft lip and/or cleft palate during the neonatal period. Turk J Med Sci 44: 696-702, 2014.

9. Fujii A, Arakaki K, Goto T, Nakama J, Tengan T, Katashima H, Shu K and Sunakawa H: Survey of lifestyle of pregnant women in Japan and Lao carrying infants with cleft lip and/or cleft palate. J Jpn Cleft Palate Assoc 37: 37-43, 2012.
10. Fulton TR, Narayanan D, Bonhoeffer J, Ortiz JR, Lambach P and Omer SB: A systematic review of adverse events following immunization during pregnancy and the newborn period. Vaccine 33: 6453-6465, 2015.

11. Yaqoob M, Mahmood F, Hanif G, Bugvi SM and Sheikh MA: Etiology and genetic factors in clefts of lip and/or palate reported at children's hospital, Lahore, Pakistan. Indian J Hum Genet 19: 136-143, 2013.

12. Li J, Guo XP, Wang KH, Zhao DH, Han T, Lang YH and Pong LJ: Correction of secondary nasal deformity of cleft lip with autogenous costal cartilage framework. Zhonghua Zheng Xing Wai Ke Za Zhi 29: 167-169, 2013 (In Chinese).

13. Go JY, Mun GH, Bang SI, Oh KS and Lim SY: The correction of alar-columella web deformities in unilateral cleft-lip nasal deformities with web graft technique. Aesthetic Plast Surg 38: 923-929, 2014

14. Henry C, Samson T and Mackay D: Evidence-based medicine: The cleft lip nasal deformity. Plast Reconstr Surg 133: 1276-1288, 2014

15. Hafezi F, Naghibzadeh B, Ashtiani AK, Mousavi SJ, Nouhi AH and Naghibzadeh G: Correction of cleft lip nose deformity with rib cartilage. Aesthet Surg J 33: 662-673, 2013.

16. Masuoka H, Kawai K, Morimoto N, Yamawaki S, Kawazoe T and Suzuki S: Conchal cartilage graft for correction of bilateral cleft lip nasal deformities during childhood. Plast Reconstr Surg Glob Open 2: e104, 2014

17. Nakamura N, Sasaguri M, Okawachi T, Nishihara K and Nozoe E: Secondary correction of bilateral cleft lip nose deformity-Clinical and three-dimensional observations on pre-and postoperative outcome. J Craniomaxillofac Surg 39: 305-312, 2011.

18. Wang Y, Shi B and Zheng Q: Efficient use of septum cartilage in correcting secondary nose deformity of unilateral cleft. Zhonghua Zheng Xing Wai Ke Za Zhi 29: 5-8, 2013 (In Chinese).

19. Park YW: Corrective rhinoplasty with combined use of autogenous auricular cartilage and porcine dermal collagen in cleft lip nose deformity. Maxillofac Plast Reconstr Surg 36: 230-236, 2014. 